\title{
Research on Pricing Strategy of Used Mobile Phone Recycling Platform
}

\author{
YanxiaGuo ${ }^{1,}$,, MingyingDong ${ }^{1}$ \\ ${ }^{1}$ School of Management, Tianjin University of Technology, Tianjin300384, China; \\ a15222839311@163.com
}

Keywords: Waste mobile phone recycling platform, Pricing strategy, Cross-group externalities, direct-group externalities, Differentiation strategy

Abstract. On the basis of two-sided markets theory and platform theory, this paper studies the pricing of waste mobile phone recycling platform from the perspective. This thesis builds models and has an analysis to the competitive platform under three kinds of charging mode, discusses the influence factors of platform pricing and profit under equilibrium state, and the pricing and profit of monopoly platform and competition platform in three kinds of charging mode, and then the model is built and analyzed in two conditions which are multi-homing and cooperation between platforms.

\section{Introduction}

With the rapid development of Internet technology and the acceleration of the renewal speed of smart mobile phone, a large amount of used mobile phones is generated in our country, which not only pollute the environment and but also is a waste of resource, and how effective and rational recycling of old mobile phones is a pressing problem. Waste recycling platform which based on mobile phone network market solve information asymmetry of conventional single market and provide an online channel for recycling waste mobile phones. How to pricing on both buyer and seller is a key question. This paper mainly studies the pricing of waste recycling mobile phone network platform. Rochet and Tirole $(2003)^{[1]}$ studied price strategy of monopoly platform in two-sided market early. They in particular offered a precise discussion of the relevant questions pertaining to such markets while Armstrong (2006) ${ }^{[2]}$ provided a very comprehensive analysis of the state of the art. Armstrong and Wright $(2007)^{[3]}$ study of the impact of the platform on both sides of goods (and services) to the platform differentiated pricing strategy. Caillaud and Jullien(2003) $)^{[4]}$ and Julian Wright ${ }^{[5]}$ respectively to the electronic media market as well as the optimal charging system credit card payment system was studied. Currently, studies on two-sided markets are mostly in Rochet and Tirole $^{[1][6]}$, Armstrong ${ }^{[2][7]}$ and Wright ${ }^{[3][5]}$ and other work-based.

This paper studies the pricing of the mobile phone network waste recycling platforms of network externalities, research ideas from Armstrong (2006) and other reference model. It will add network externalities to the utility function of waste mobile phone recycling platform and will propose optimal pricing strategies among the following three charging modes.

\section{The pricing strategy of competitive Platform}

In real life, most of the waste mobile phone network recovery platform exists in the form of competitive, On the basis of this, this paper considers the competition between two waste mobile phone network recovery platforms, which adopt the same pricing method and pricing formula.

Assume that the market has two waste recycling mobile phone network platforms. Both platforms are located at both ends of the line, and were evenly distributed on the user line segment, $d_{l}(l=1,2)$ represents the platform differences. The number of recyclers and consumers are $n_{b}$ and $n_{s}$ respectively, the utility of the buyer and seller in the platform obtained are $u_{b}^{i}$ and $u_{s}^{i}$ respectively. The number of users are 1, according to the classic Hotelling Model, the number of users of both the two platforms can be traded separately as: 


$$
n_{b}^{i}=\frac{1}{2}+\frac{u_{b}^{i}-u_{b}^{j}}{2 d_{1}}, n_{s}^{i}=\frac{1}{2}+\frac{u_{s}^{i}-u_{s}^{j}}{2 d_{2}}(i, j=1,2)
$$

Next, we will discuss the pricing strategy under three circumstances of competitive platform.

Competition between two platforms on registration fees. According to Armstrong in both sides of the single ownership of the two registration fee for a competitive platform for discussion, the two sides of the user on the different platforms of the utility function can be expressed as:

$$
u_{b}^{i}=\alpha_{b} n_{s}^{i}-\beta_{b} n_{b}^{i}-p_{\mathrm{rb}}^{i}, \quad u_{s}^{i}=\alpha_{s} n_{b}^{i}+\beta_{s} n_{s}^{i}-p_{\mathrm{rs}}^{i}
$$

Take the Eq.2 into the formula Eq.1, the share of platform $i$ in the recovery of business and consumer market respectively:

$$
n_{b}^{i}=\frac{1}{2}+\frac{\alpha_{b}\left(2 n_{s}^{i}-1\right)+p_{b}^{j}-p_{b}^{i}}{2\left(d_{1}+\beta_{b}\right)}, n_{s}^{i}=\frac{1}{2}+\frac{\alpha_{s}\left(2 n_{b}^{i}-1\right)+p_{s}^{j}-p_{s}^{i}}{2\left(d_{2}-\beta_{s}\right)}
$$

The above two equations can be obtained simultaneously:

$$
n_{b}^{i}=\frac{1}{2}+\frac{\alpha_{b}\left(p_{r s}^{j}-P_{r s}^{i}\right)+\left(d_{2}-\beta_{s}\right)\left(p_{r b}^{j}-p_{r b}^{i}\right)}{2\left[\left(d_{1}+\beta_{b}\right)\left(d_{2}-\beta_{s}\right)-\alpha_{b} \alpha_{s}\right]}, \quad n_{s}^{i}=\frac{1}{2}+\frac{\alpha_{s}\left(p_{r b}^{j}-p_{r b}^{i}\right)+\left(d_{1}+\beta_{b}\right)\left(p_{r s}^{j}-p_{r s}^{i}\right)}{2\left[\left(d_{1}+\beta_{b}\right)\left(d_{2}-\beta_{s}\right)-\alpha_{b} \alpha_{s}\right]}
$$

The profit function of platform can be expressed as:

$$
\pi^{i}=\left(p_{\mathrm{rb}}^{i}-f_{b}\right) n_{b}^{i}+\left(p_{\mathrm{rs}}^{i}-f_{s}\right) n_{s}^{i}
$$

The necessary and sufficient condition for the research of the bilateral market from the Armstrong to the market distribution equilibrium is $4 d_{1} d_{2}>\left(\alpha_{b}+\alpha_{s}\right)^{2}$. Assuming that the platform reach a state of equilibrium at this time, that is $p_{\mathrm{rb}}^{1}=p_{\mathrm{rb}}^{2}=p_{\mathrm{rb}}, p_{\mathrm{rs}}^{1}=p_{\mathrm{rs}}^{2}=p_{\mathrm{rs}}$, then derivation of a $p_{\mathrm{rb}}$, , $p_{\mathrm{rs}}$ respectively, get the price equilibrium solution:

$$
p_{\mathrm{r} b}=f_{\mathrm{b}}+d_{1}-\alpha_{\mathrm{s}}+\beta_{\mathrm{b}}, \quad p_{\mathrm{rs}}=f_{\mathrm{s}}+d_{2}-\alpha_{b}-\beta_{\mathrm{s}}
$$

Single platform profit is:

$$
\pi=\left(d_{1}+d_{2}-\alpha_{b}-\alpha_{s}-\beta_{s}+\beta_{b}\right) / 2
$$

Both sides of the users of consumer surplus:

$$
n_{b}^{1} u_{b}^{1}+n_{b}^{2} u_{b}^{2}+n_{s}^{1} u_{s}^{1}+n_{s}^{2} u_{s}^{2}=3\left(\alpha_{b}+\alpha_{s}-\beta_{b}+\beta_{s}\right) / 2-\left(f_{b}+f_{s}\right)-\left(d_{1}+d_{2}\right)
$$

Total social welfare:

$$
w=\left(\alpha_{b}+\alpha_{s}-\beta_{b}+\beta_{s}\right) / 2-\left(f_{b}+f_{s}\right)
$$

The competition between the two platforms on transaction fee. The utility function for the seller and the buyer on both sides of the platform is:

$$
u_{b}^{i}=\alpha_{b} n_{s}^{i}-\beta_{b} n_{b}^{i}-p_{t b}^{i}\left(t_{1} / 2\right), \quad u_{s}^{i}=\alpha_{s} n_{b}^{i}+\beta_{s} n_{s}^{i}-p_{t b}^{i}\left(t_{2} / 2\right)
$$

The Eq.4 into the Eq.1 can be recovered in platform and the consumer market share respectively:

$$
\begin{aligned}
& n_{b}^{i}=\frac{1}{2}+\frac{\alpha_{b} \mathrm{t}_{2}\left(p_{r s}^{j}-p_{r s}^{i}\right)+\mathrm{t}_{1}\left(d_{2}-\beta_{s}\right)\left(p_{r b}^{j}-p_{r b}^{i}\right)}{4\left[\left(d_{1}+\beta_{b}\right)\left(d_{2}-\beta_{s}\right)-\alpha_{b} \alpha_{s}\right]} \\
& n_{s}^{i}=\frac{1}{2}+\frac{\alpha_{s} \mathrm{t}_{1}\left(p_{r b}^{j}-p_{r b}^{i}\right)+\mathrm{t}_{2}\left(d_{1}+\beta_{b}\right)\left(p_{r s}^{j}-p_{r s}^{i}\right)}{4\left[\left(d_{1}+\beta_{b}\right)\left(d_{2}-\beta_{s}\right)-\alpha_{b} \alpha_{s}\right]}
\end{aligned}
$$


The profit function of the platform $i$ is:

$$
\pi^{i}=\left[\left(p_{\mathrm{tb}}^{i}-c_{\mathrm{b}}\right)\left(t_{1} / 2\right) \lambda n_{b}^{i}+\left(p_{t s}^{i}-c_{\mathrm{b}}\right)\left(t_{2} / 2\right) \lambda n_{s}^{i}\right] / 2
$$

Assuming two platforms for the same side of the user to charge the same transaction fees, that is $p_{t b}^{1}=p_{t b}^{2}=p_{t b}, p_{t s}^{1}=p_{t s}^{2}=p_{t s}$, respectively $p_{\mathrm{rb}}, p_{\mathrm{rs}}$ the derivation, get the price equilibrium solution:

$$
p_{t b}=c_{\mathrm{b}}+\frac{2}{t}\left(d_{1}-\alpha_{s}+\beta_{b}\right), \quad p_{t s}=c_{\mathrm{s}}+\frac{2}{t}\left(d_{2}-\alpha_{b}-\beta_{s}\right)
$$

Single platform profit is:

$$
\pi=\lambda\left(d_{1}+d_{2}-\alpha_{b}-\alpha_{s}-\beta_{s}+\beta_{b}\right) / 4
$$

In the equilibrium state, for the two competitive mobile phone network platform in terms of waste recycling, the profit from the transaction fee is less than the profit from the registration fee. So, waste recycling platform of mobile phone network tends to charge the registration fee to obtain greater profits.

Both sides of the users of consumer surplus platform:

$$
n_{b}^{1} u_{b}^{1}+n_{b}^{2} u_{b}^{2}+n_{s}^{1} u_{s}^{1}+n_{s}^{2} u_{s}^{2}=3\left(\alpha_{b}+\alpha_{s}-\beta_{b}+\beta_{s}\right) / 2-t\left(c_{1}+c_{2}\right) / 2-\left(d_{1}+d_{2}\right)
$$

Total social welfare:

$$
w=\frac{3-\lambda}{2}\left(\alpha_{b}+\alpha_{s}-\beta_{b}+\beta_{s}\right)-\frac{2-\lambda}{2}\left(d_{1}+d_{2}\right)-\frac{t}{2}\left(c_{1}+c_{2}\right)
$$

Using two step plan on both sides. The utility function that two-side users expected is:

$$
u_{b}^{i}=\alpha_{b} n_{s}^{i}-\beta_{b} n_{b}^{i}-p_{r b}^{i}-p_{t b}^{i}\left(t_{1} / 2\right), \quad u_{s}^{i}=\alpha_{s} n_{b}^{i}+\beta_{s} n_{s}^{i}-p_{r b}^{i}-p_{t b}^{i}\left(t_{2} / 2\right)
$$

Take Eq.5 to theEq.1, then can get the market share of recycling business and consumers respectively:

$$
\begin{aligned}
& n_{b}^{i}=\frac{1}{2}+\frac{1}{2} \frac{\alpha_{b}\left[p_{r s}^{j}-p_{r s}^{i}+\left(\mathrm{t}_{2} / 2\right)\left(p_{t s}^{j}-p_{t s}^{i}\right)\right]+\left(d_{2}-\beta_{s}\right)\left[p_{r b}^{j}-p_{r b}^{i}+\left(\mathrm{t}_{1} / 2\right)\left(p_{t b}^{j}-p_{t b}^{i}\right)\right]}{\left(d_{1}+\beta_{b}\right)\left(d_{2}-\beta_{s}\right)-\alpha_{b} \alpha_{s}} \\
& n_{s}^{i}=\frac{1}{2}+\frac{1}{2} \frac{\alpha_{s}\left[p_{r b}^{j}-p_{r b}^{i}+\left(\mathrm{t}_{1} / 2\right)\left(p_{t b}^{j}-p_{t b}^{i}\right)\right]+\left(d_{1}+\beta_{b}\right)\left[p_{r s}^{j}-p_{r s}^{i}+\left(\mathrm{t}_{2} / 2\right)\left(p_{t s}^{j}-p_{t s}^{i}\right)\right]}{\left(d_{1}+\beta_{b}\right)\left(d_{2}-\beta_{s}\right)-\alpha_{b} \alpha_{s}}
\end{aligned}
$$

The profit function of platform is:

$$
\pi^{i}=\left(p_{\mathrm{rb}}^{i}-f_{b}\right) n_{b}^{i}+\left(p_{\mathrm{rs}}^{i}-f_{s}\right) n_{s}^{i}+\left[\left(p_{\mathrm{tb}}^{i}-c_{\mathrm{b}}\right)\left(t_{1} / 2\right) \lambda n_{b}^{i}+\left(p_{t s}^{i}-c_{\mathrm{b}}\right)\left(t_{2} / 2\right) \lambda n_{\mathrm{s}}^{i}\right] / 2
$$

Assuming that the platform to achieve the equilibrium state, that is, $p_{\mathrm{rb}}^{1}=p_{\mathrm{rb}}^{2}=p_{\mathrm{r} b}$, $p_{\mathrm{rs}}^{1}=p_{\mathrm{rs}}^{2}=p_{\mathrm{rs}}, \quad p_{\mathrm{tb}}^{1}=p_{\mathrm{tb}}^{2}=p_{\mathrm{tb}}, \quad p_{\mathrm{ts}}^{1}=p_{\mathrm{ts}}^{2}=p_{\mathrm{ts}}$, can get the two platform trading fee pricing formula:

$$
p_{\mathrm{r} b}=f_{\mathrm{b}}+d_{1}-\alpha_{\mathrm{s}}+\beta_{b}-\frac{\lambda t}{4}\left(p_{\mathrm{tb}}-c_{\mathrm{b}}\right), \quad p_{\mathrm{rs}}=f_{\mathrm{s}}+d_{2}-\alpha_{b}-\beta_{\mathrm{s}}-\frac{\lambda t}{4}\left(p_{t s}-c_{\mathrm{s}}\right)
$$

Single platform profit:

$$
\pi=\left(d_{1}+d_{2}-\alpha_{b}-\alpha_{s}-\beta_{s}+\beta_{b}\right) / 2
$$

We can see that, in the equilibrium state, the two step charging system and the only collection of registration fees are more than the profit margin is only a transaction time

Both sides of the users of consumer surplus:

$$
n_{b}^{1} u_{b}^{1}+n_{b}^{2} u_{b}^{2}+n_{s}^{1} u_{s}^{1}+n_{s}^{2} u_{s}^{2}=\frac{3}{2}\left(\alpha_{b}+\alpha_{s}-\beta_{b}+\beta_{s}\right)-\left(d_{1}+d_{2}\right)-\left(f_{b}+f_{s}\right)-\frac{(2-\lambda) t}{4}\left(p_{t b}+p_{t s}\right)-\frac{\lambda t}{4}\left(c_{1}+c_{2}\right)
$$


Total social welfare:

$$
w=\frac{1}{2}\left(\alpha_{b}+\alpha_{s}-\beta_{b}+\beta_{s}\right)-\left(f_{b}+f_{s}\right)-\frac{(2-\lambda) t}{4}\left(p_{t b}+p_{t s}\right)-\frac{\lambda t}{4}\left(c_{1}+c_{2}\right)
$$

We can see that, two competitive waste mobile phone network recovery platform in the case of taking two steps, the difference degree of the platform, the cross network externalities between the two sides and the direct network externalities between the same side users are similar to the effect of the profit and welfare.

\section{Summary}

Through analysis of the three mobile network fee systems in the competitive waste recycling mobile phone network platform, we can draw the following conclusions:

(1)In the study of two-sided market, the pricing of the platform will be affected by the degree of differentiation, the cross network externalities and the direct network externalities.

(2)For the competitive platform, in the above three different charging mode, increase the selection differentiation and improve the recycling business between the competition will increase the profit of the platform; increase the cross network externalities and the demonstration effect will decrease the profit of the platform.

\section{References}

[1] Rochet J C, Tirole J. Platform competition in two-sided markets[J]. Journal of the European Economic Association, 2003, 1(4): 990-1029.

[2] Mark Armstrong. Competition in two-sided markets[J].Journal of Economics, 2006, 37(3): 668-691.

[3] Armstrong M, Wright J. Two-sided markets, competitive bottlenecks and exclusive contracts[J]. Economic Theory, 2007, 32(2): 353-380.

[4] Caillaud B, Jullien B. Chicken \& egg: Competition among intermediation service providers[J]. Randjournal of Economics, 2003: 309-328.

[5] Julian Wright. Optimal card payment systems[J]. European Economic Review, 2003, 47: 587-612.

[6] Rochet J C, Tirole J. Two-sided markets: A progress report[J]. Rand Journal of Economics, 2006, 37(3): 645-667.

[7] Armstrong M. Two-sided markets: economic theory and policy implications[J]. Recent Developments in Antitrust: Theory and Evidence, 2007: 39-59. 